Y. Furuta

Nagoya Math. J.

Vol. 62 (1976), 13-28

\title{
ON NILPOTENT FACTORS OF CONGRUENT IDEAL CLASS GROUPS OF GALOIS EXTENSIONS
}

\author{
YOSHIOMI FURUTA
}

\section{Introduction.}

Let $K$ be a Galois extension of an algebraic number field $k$ of finite degree with Galois group g. Then $g$ acts on a congruent ideal class group $\mathfrak{S}$ of $K$ as a group of automorphisms, when the class field $M$ over $K$ corresponding to $\mathfrak{D}$ is normal over $K$. Let $I_{g}$ be the augmentation ideal of the group ring $Z \mathrm{~g}$ over the ring of integers $Z$, namely $I_{\mathrm{g}}$ be the ideal of $Z \mathfrak{g}$ generated by $\sigma-1, \sigma$ running over all elements of g. Then $I_{8}$ ? is the group of all elements $\mathfrak{a}^{\sigma-1}$ where $a$ and $\sigma$ belong to (D) and $g$ respectively. Put $I_{g}^{i+1} \mathfrak{D}=I_{g}\left(I_{\mathfrak{g}}^{i} \mathfrak{D}\right)$ for $i=0,1,2, \ldots$. Then we have the sequence $\mathfrak{D} \supset I_{8} \mathfrak{D} \supset I_{8}^{2} \mathfrak{D} \supset \ldots$ and call it the lower central series for $\mathfrak{D}$ with respect to $\mathrm{g}$.

Denote by $K_{M / k}^{(i)}$ or simply by $K^{(i)}$ the class field over $K$ corresponding to $I_{\mathfrak{g}}^{i \mathfrak{D}}$ and denote by $G\left(K^{(i+1)} / K^{(i)}\right)$ the Galois group of $K^{(i+1)}$ over $K^{(i)}$. Then for $i=1$ the field $K^{(1)}$ is called the central class field of $K$ in $M$ with respect to $k$, and some structure of $G\left(K^{(1)} / K\right)$ has been studied in [5] and [6], when $M$ is the absolute class field of $K$.

The purpose of the present paper is to investigate the structure of the lower central series for $\mathfrak{D}$ or the structure of the Galois groups $G\left(K^{(i+1)} / K^{(i)}\right)$ for $i=0,1,2, \cdots$.

When $K$ is a quadratic extension of the rational number field, the explicit criteria for the divisibility of the class number by power of 2 has been studied by various authors. Especially P. Barrucand and H. Cohn [2] and H. Hasse [9] gave new criteria resently, and G. Gras [7] and [8] studied the structure of $\ell$-class groups of ideals for cyclic extensions of degree a prime $\ell$. The foundation of the argument was a generalization of the ambigous class. This can be considered as a study

Received September 22, 1975.

Revised February 2, 1976. 
of the "upper" central series for $\mathscr{D}$ in the above sense when $\mathscr{D}$ is the absolute ideal class group of a cyclic extension $K$ over $k$ of a prime degree.

In the present paper, we shall see that the investigation of the "lower" central series make simplify the argument and possible to generalize it to the case of non-cyclic Galois extensions. In $\S 1$ we treat the lower central series for the ideal class group of cyclic extensions and it is reduced to the structure in the genus group. In the case of cyclic extensions our argument is quite simple, but it is not so in the case of non-cyclic extensions though the result is close to that of cyclic case (Remark to Theorem 5). This is caused by the fact that the augmentation ideal $I_{g}$ operates on $D$ as a homomorphism and the homomorphism theorem can be used in the case of cyclic extensions, but this does not hold in the case of non-cyclic extensions. Our main purpose in $\S 2$ below is to reduce the structure of $G\left(K^{(i+1)} / K^{(i)}\right)$ to the structure in the central class group $G\left(K^{(1)} / K\right)$ which coincides with the genus group in the case of cyclic extensions. First of all in $\S 2$ we recall the structure of $G\left(K^{(1)} / K\right)$ in general case. Namely, the argument of the genus field and central class field for the absolute class field, which has been treated in our previous papers [4], [5] and [6], is generalized to that for any class fields. In $\S 3$ we study on cohomological expressions of central class groups (Theorem 1 and 2), and in $\S 4$ we express $G\left(K^{(i+1)} / K^{(i)}\right)$ by cohomology groups attached to $K / k$ (Theorem 3 and 4 ). Then in $\S 5$ we have the main result (Theorem 5).

\section{§1. The case of cyclic extensions.}

Let $k$ be an algebraic number field of finite degree and $K$ be a cyclic extension of finite degree with Galois group $g$ generated by $\sigma$. Moreover let $M$ be a class field over $K$ corresponding to the congruent ideal class group $\mathfrak{D}$. We assume that $M$ is normal over $k$ and we define an endomorphism $\varphi^{i}$ on $\subseteq$ by $\varphi^{i}(\mathfrak{a})=\mathfrak{a}^{(\sigma-1)^{i}}$ for any $\mathfrak{a}$ of $\mathfrak{D}$ and $i=$ $1,2, \cdots$. Then we see $I_{g}^{i \mathfrak{D}}=\varphi^{i}(\mathfrak{D})$. Let $K^{(i)}$ be the class field over $K$ corresponding to $\varphi^{i}(\mathfrak{D})$ and denote by $G\left(K^{(i+1)} / K^{(i)}\right)$ the Galois group of $K^{(i+1)}$ over $K^{(i)}$. Then we have

$$
G\left(K^{(i+1)} / K^{(i)}\right) \cong \varphi^{i}(\mathfrak{D}) / \varphi^{i+1}(\mathfrak{D}) .
$$

Let $\overline{\varphi^{i}}$ be the homomorphism of $\mathscr{D} / \varphi(\mathfrak{D})$ to $\varphi^{i}(\mathfrak{D}) / \varphi^{i+1}(\mathfrak{D})$ induced from $\varphi^{i}$ 
and denote by $N^{(i)}$ the kernel of $\varphi^{i}$. Then the kernel of $\overline{\varphi^{i}}$ is equal to $N^{(i)} \varphi(\mathfrak{D}) / \varphi(\mathfrak{D})$, and we have

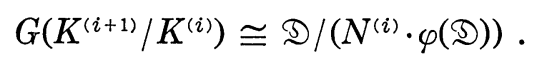

When $M$ is the absolute class field, $K^{(1)}$ is the genus field of $K$ with respect to $k$ and the structure of the group $G\left(K^{(1)} / K\right)$, which is isomorphic to $\mathfrak{D} / \varphi(\mathfrak{D})$, is known largely ${ }^{1}$. We are able to study the structure of $G\left(K^{(i+1)} / K^{(i)}\right)$ more explicitly by means of this way, for which we will treat in other paper. In the following sections we shall study to obtain a corresponding formula to (1) in the case where $K$ is not necessarily cyclic over $k$.

\section{§2. The genus group and the central class group.}

For any algebraic number field $K$ we denote by $J_{K}$ and $K^{\times}$the idele group of $K$ and the multiplicative group of non-zero elements of $K$ which is embedded in $J_{K}$ in usual way. For an extension $L$ of $K$ of finite degree we denote by $N_{L / K}$ the norm from $L$ to $K$ and by $(L: K)$ the extension degree. When $L$ is normal over $K$, we denote by $G(L / K)$ the Galois group of $L$ over $K$.

Let $M \supset K \supset k$ be a sequence of extensions of algebraic number fields of finite degree. Denote by $K_{M / k}^{*}$ the maximal extension of $K$ which is contained in $M$ and is obtained from $K$ by composing an abelian extension over $k . K_{M / k}^{*}$ is called the genus field of $K$ in $M$ with respect to $k$. When $M$ is the absolute class field $\bar{K}$ of $K, K_{\bar{K} / k}^{*}$ is called ${ }^{2)}$ simply the genus field of $K$ with respect to $k$.

Proposition $1^{3)}$. Let notation be as above. Then $K_{M / k}^{*}$ is normal over $K$ and we have

$$
G\left(K_{M / k}^{*} / K\right) \cong \frac{N_{K / k} J_{K}}{N_{K / k} J_{K} \cap k^{\times} N_{M / k} J_{M}}
$$

Proof. Let $M_{0}$ and $K_{0}$ be the maximal abelian extensions over $k$ contained in $M$ and $K$ respectively. Then $K_{M / k}^{*}=K M_{0}$ and the transfer theorem of class field theory implies $G\left(K_{M / k}^{*} / K\right) \cong G\left(M_{0} / K_{0}\right) \cong k^{\times} N_{K_{0} / k} J_{K_{0}}$ $/ k^{\times} N_{M_{0} / k} J_{M_{0}} \cong k^{\times} N_{K / k} J_{K} / k^{\times} N_{M / k} J_{M} \cong N_{K / k} J_{K} /\left(N_{K / k} J_{K} \cap k^{\times} N_{M / k} J_{M}\right)$.

1) Cf. Furuta [4].

2) Cf. Fröhlich [3].

3) Cf. Furuta [4]. 
Let $M \supset K \supset k$ be as above and $\mathfrak{p}$ be an any prime of $M$. For the prime of $K$ or $M$ which is divisible by $\mathfrak{p}$, we use the same letter $\mathfrak{p}$ for the sake of simplicity.

We call $M$ an $E L$-genus extension ${ }^{4)}$ of $K$ with respect to $k$ if $M_{\mathfrak{p}}$ is obtained from $K_{\mathfrak{p}}$ by composing an abelian extension over $k_{\mathfrak{p}}$ for every prime $\mathfrak{p}$.

We call $L$ a central extension of $K$ with respect to $k$, if $L$ is an extension of $K$ which is normal over $k$ and $G(L / K)$ is contained in the center of $G(L / k)$.

Now for a sequence $M \supset K \supset k$, we denote by $\hat{K}_{M / k}$ the maximal extension of $K$ which is $E L$-genus and central with respect to $k$ and is contained in $M$. When $M$ is the absolute class field $\bar{K}$ of $K, \hat{K}_{\bar{K} / k}$ is called the central class field ${ }^{5)}$ of $K$ with respect to $k$.

Proposition 2 (Masuda [11]). Notation being as above, we have

$$
G\left(\hat{K}_{M / k} / K\right) \cong N_{K / k} J_{K} /\left(N_{K / k} K^{\times} \cdot N_{M / k} J_{M}\right) \text {. }
$$

Combining with Proposition 1 we have ${ }^{6)}$ the following

Proposition 3. Notation being as above,

$$
G\left(\hat{K}_{M / k} / K_{M / k}^{*}\right) \cong\left(k^{\times} \cap N_{K / k} J_{K}\right) /\left(N_{K / k} K^{\times} \cdot\left(k^{\times} \cap N_{M / k} J_{M}\right)\right) .
$$

When $M$ is abelian over $K$, we have further the following

Proposition 4. Let $K$ be an extension of $k$ and $M$ be an abelian extension of $K$. Let $L$ be a subfield of $M$ and assume that $L$ contains $K_{M / k}^{*}$. Then we have

$$
G\left(\hat{L}_{M / k} / L\right) \cong \frac{k^{\times} \cap N_{L / k} J_{L}}{N_{K / k}\left(K^{\times} \cap N_{L / K} J_{K}\right)\left(k^{\times} \cap N_{M / k} J_{M}\right)} .
$$

Proof. Proposition 2 implies

$$
G\left(\hat{L}_{M / k} / L\right) \cong N_{L / k} J_{L} / N_{L / k}\left(L^{\times} N_{M / L} J_{M}\right)
$$

Moreover by the translation theorem in class field theory,

$$
N_{L / k}\left(L^{\times} N_{M / L} J_{M}\right)=N_{K / k}\left(N_{L / K}\left(L^{\times} \cdot N_{M / L} J_{M}\right)\right)
$$

4) Cf. Masuda [11], in which this is called an EL-abelian extension.

5) Cf. Furuta [5]. In the case where $M=\bar{K}, M$ itself is already EL-genus, because $\bar{K}$ is unramified extension over $k$.

6) Cf. Furuta $[5$, p.151]. 


$$
=N_{K / k}\left(K^{\times} N_{M / K} J_{M} \cap N_{L / K} J_{L}\right)
$$

Hence we have

$$
N_{L / k}\left(L^{\times} N_{M / L} J_{M}\right)=N_{K / k}\left(K^{\times} \cap N_{L / K} J_{L}\right) \cdot N_{M / k} J_{M} .
$$

On the other hand $L$ contains $K_{M / k}^{*}=K M_{0}$, where $M_{0}$ is the maximal abelian extension of $k$ contained in $M$. Hence $L{ }_{M / k}^{*}=L M_{0}=L$, and Proposition 1 implies

$$
N_{L / k} J_{L}=N_{L / k} J_{L} \cap k^{\times} N_{M / k} J_{M}=\left(k^{\times} \cap N_{L / k} J_{L}\right) \cdot N_{M / k} J_{M} .
$$

Thus we have

$$
\begin{aligned}
G\left(\hat{L}_{M / k} / L\right) & \cong \frac{\left(k^{\times} \cap N_{L / k} J_{L}\right) \cdot N_{M / k} J_{M}}{N_{K / k}\left(K^{\times} \cap N_{L / k} J_{L}\right) \cdot N_{M / k} J_{M}} \\
& \cong \frac{k^{\times} \cap M_{L / k} J_{L}}{N_{K / k}\left(K^{\times} \cap N_{L / K} J_{L}\right) \cdot\left(k^{\times} \cap N_{M / K} J_{M}\right)}
\end{aligned}
$$

Let us consider the special case where $M$ is the absolute class field of $K$, which we denote by $\bar{K}$. Let $U_{K}$ be the unit idele group of $K$ whose real infinite components are the group of all non-zero real numbers or of all positive real numbers according as we treat $\bar{K}$ in wide sense $^{7)}$ or in narrow sense.

Proposition 5. Let $K$ be a Galois extension of $k$ and $L$ be a subfield of the absolute class field $\bar{K}$ of $K$. Assume that $L$ contains the genus field $K^{*}$ of $K$ with respect to $k$ and $L$ is normal over $k$. Put $G=G(L / k)$ and $H=G(L / K)$. Then we have

$$
G\left(\hat{L}_{\bar{K} / k} / L\right) \cong \frac{k^{\times} \cap N_{L / k} J_{L}}{N_{K / k}\left(K^{\times} \cap N_{L / K} J_{L}\right)\left(E_{k} \cap N_{K / k} U_{K}\right)},
$$

where $E_{k}$ stands for the global unit group of $K$ which is embedded in $J_{k}$ in usual way.

Proof. We have $K^{\times} N_{M / K} J_{M}=K^{\times} N_{\bar{K} / K} J_{\bar{K}}=K^{\times} U_{K}$ and $N_{L / k} J_{L} \supset$ $N_{K / k} U_{K}$, since $L$ is unramified over $K$. Hence the formulas (2) and (3) in the proof of Proposition 4 are replaced by

$$
\begin{aligned}
N_{L / k}\left(L^{\times} N_{M / L} J_{M}\right) & =N_{K / k}\left(K^{\times} U_{K} \cap N_{L / K} J_{L}\right) \\
& =N_{K / k}\left(K^{\times} \cap N_{L / K} J_{L}\right) \cdot N_{K / k} U_{K}
\end{aligned}
$$

7) This means that all infinite primes are not ramified too. 
and

$$
\begin{aligned}
N_{L / k} J_{L} & =N_{L / k} J_{L} \cap k^{\times} N_{\bar{K} / k} J_{\bar{K}}=N_{L / k} J_{L} \cap k^{\times} \cdot N_{K / k}\left(K^{\times} N_{\bar{K} / K} J_{\bar{K}}\right) \\
& =N_{L / k} J_{L} \cap k^{\times} N_{K / k}\left(K^{\times} U_{K}\right)=\left(k^{\times} \cap N_{L / k} J_{L}\right) \cdot N_{K / k} U_{K} .
\end{aligned}
$$

Thus the formula (4) is also replaced by

$$
\begin{aligned}
G\left(\hat{L}_{\bar{K} / k} / L\right) & \cong \frac{\left(k^{\times} \cap N_{L / k} J_{L}\right) \cdot N_{K / k} U_{K}}{N_{K / k}\left(K^{\times} \cap N_{L / K} J_{L}\right) \cdot N_{K / k} U_{K}} \\
& \cong \frac{k^{\times} \cap N_{L / k} J_{L}}{N_{K / k}\left(K^{\times} \cap N_{L / K} J_{L}\right)\left(k^{\times} \cap N_{K / k} U_{K}\right)}
\end{aligned}
$$

and the proposition follows.

\section{§3. Cohomological expression of $G\left(\hat{L}_{M / k} / L\right)$.}

Let $K$ be a Galois extension of $k$ and $M$ be an abelian extension of $K$. Let further $L$ be a subfield of $M$. Assume that $L$ contains the genus field $K_{M / k}^{*}$ and $L$ is normal over $k$. Put $G=G(L / k), H=G(L / K)$ and $C_{L}=J_{L} / L^{\times}$.

We consider a natural exact sequence

$$
0 \longrightarrow L^{\times} \stackrel{i}{\longrightarrow} J_{L} \stackrel{j}{\longrightarrow} C_{L} \longrightarrow 0 \text {. }
$$

Then we have the following commutative diagram, where the rows are exact and the columns are corestrictions $\operatorname{Cor}_{H, G}$ :

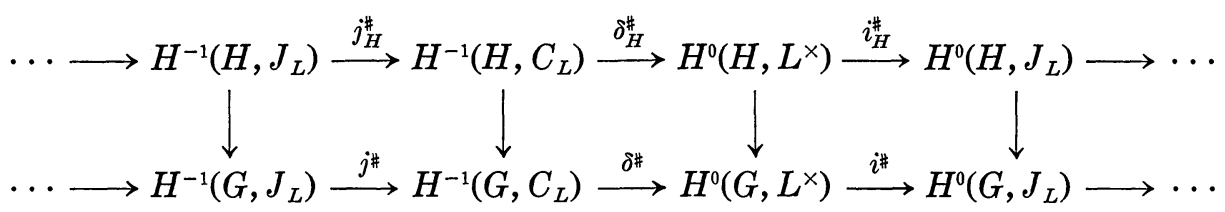

Let $f$ be a natural homomorphism of $H^{0}\left(G, L^{\times}\right)$to $H^{0}\left(G, L^{\times}\right)$/ $\operatorname{Cor}_{H, G} \delta_{H}^{\sharp} H^{-1} \cdot\left(H, C_{L}\right)$.

Now we put

$$
\left\{\begin{array}{l}
X=\left(k^{\times} \cap N_{L / k} J_{L}\right) / N_{K / k}\left(K^{\times} \cap N_{L / K} J_{L}\right), \\
Y=N_{K / k}\left(K^{\times} \cap N_{L / K} J_{L}\right)\left(k^{\times} \cap N_{M / k} J_{M}\right) / N_{K / k}\left(K^{\times} \cap N_{L / K} J_{L}\right) .
\end{array}\right.
$$

Then by Proposition 4 we have

$$
G\left(\hat{L}_{M / k} / L\right) \cong X / Y
$$

For any finite group $G$ and any $G$-module $A$ we denote by $\kappa_{0}$ the standard 
isomorphism of $H^{0}(G, A)$ to $A^{G} / N_{G} A$, where $A^{G}$ is the subgroup of $A$ consisting of all $G$-invariant elements and $N_{G}$ is the trace map. Then by $\kappa_{0}$ we have

$$
\begin{aligned}
N_{K / k}\left(K^{\times} \cap N_{L / K} J_{L}\right) / N_{K / k}\left(N_{L / K} L^{\times}\right) & \cong \operatorname{Cor}_{H, G}\left(\operatorname{Ker} i_{H}^{\#}\right) \\
& \cong \operatorname{Cor}_{H, G} \delta_{H}^{\sharp} H^{-1}\left(H, C_{L}\right)
\end{aligned}
$$

and moreover

$$
\begin{aligned}
X & \cong\left(\left(k^{\times} \cap N_{L / k} J_{L}\right) / N_{L / k} L^{\times}\right) /\left(N_{K / k}\left(K^{\times} \cap N_{L / K} J_{L}\right) / N_{K / k}\left(N_{L / K} L^{\times}\right)\right) \\
& \cong f \cdot \delta^{\sharp} H^{-1}\left(G, C_{L}\right)=\delta^{\sharp} H^{-1}\left(G, C_{L}\right) / \operatorname{Cor}_{H, G} \delta_{H}^{\sharp} H^{-1}\left(H, C_{L}\right) \\
& \cong \delta^{\sharp} H^{-1}\left(G, C_{L}\right) / \delta^{\sharp} \operatorname{Cor}_{H, G} H^{-1}\left(H, C_{L}\right) \\
& \cong H^{-1}\left(G, C_{L}\right) /\left(\operatorname{Cor}_{H, G} H^{-1}\left(H, C_{L}\right)+\operatorname{Ker} \delta^{\sharp}\right) .
\end{aligned}
$$

Since $\operatorname{Ker} \delta^{\sharp}=j^{\sharp} H^{-1}\left(G, J_{L}\right)$, we have

$$
X \cong \frac{H^{-1}\left(G, C_{L}\right)}{\operatorname{Cor}_{H, G} H^{-1}\left(H, C_{L}\right)+j^{\sharp} H^{-1}\left(G, J_{L}\right)} .
$$

Next, we translate $Y$ on the same stage for $X$. Consider the following commutative diagram whose rows and columns are exact by the natural homomorphisms:

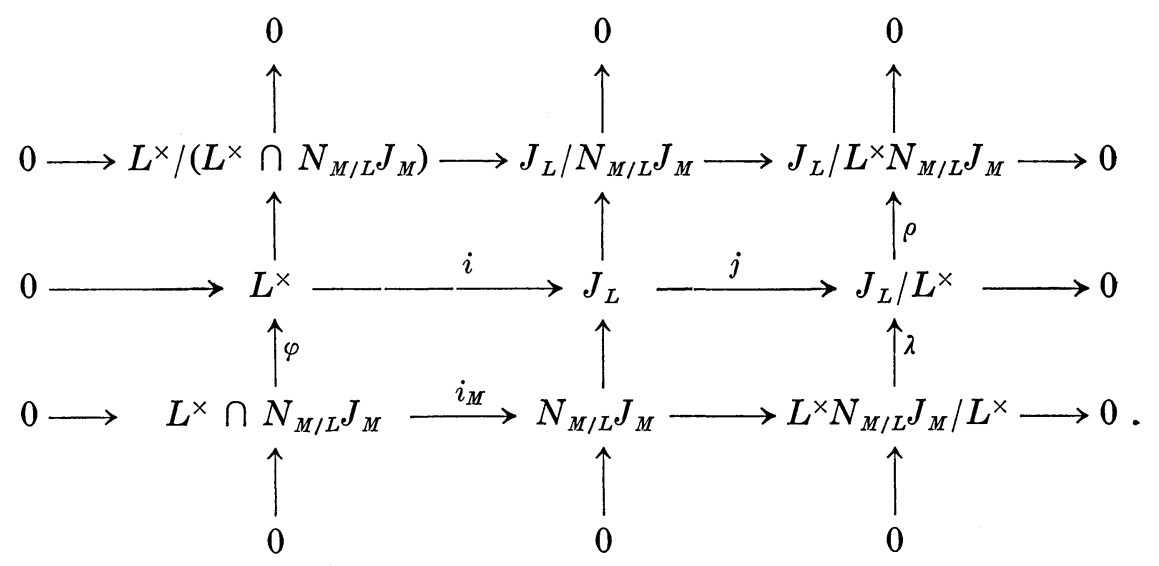

Then we have the following commutative cohomology exact sequence. 


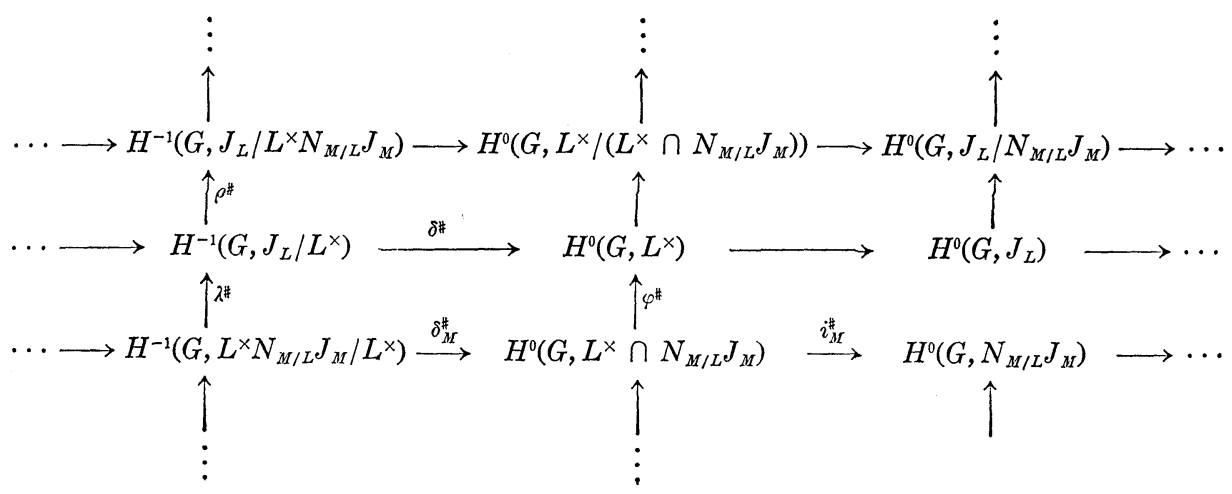

This implies that

$$
\begin{aligned}
& \left(k^{\times} \cap N_{M / k} J_{M}\right) / N_{L / k}\left(L^{\times} \cap N_{M / L} J_{M}\right) \\
& \quad=\left(\left(L^{\times} \cap N_{M / L} J_{M}\right)^{G} \cap N_{M / k} J_{M}\right) / N_{L / k}\left(L^{\times} \cap N_{M / L} J_{M}\right) \\
& \cong \operatorname{Ker} i_{M}^{\#}=\delta_{M}^{\#} H^{-1}\left(G, L^{\times} N_{M / L} J_{M} / L^{\times}\right) .
\end{aligned}
$$

Moreover since $\left(\left(L^{\times} \cap N_{M / L} J_{M}\right)^{G} \cap N_{K / k}\left(K^{\times} \cap N_{L / K} J_{L}\right)\right) / N_{L / k}\left(L^{\times} \cap N_{M / L} J_{M}\right)$ $=\operatorname{Ker}\left(f \circ \varphi^{\sharp}\right)$ by (7), we have $\left(\left(k^{\times} \cap N_{M / k} J_{M}\right) \cap N_{K / k}\left(K^{\times} \cap N_{L / K} J_{L}\right)\right) /$ $N_{L / k}\left(L^{\times} \cap N_{M / L} J_{M}\right)=\delta_{M}^{\#} H^{-1}\left(G, L^{\times} N_{M / L} J_{M} / L^{\times}\right) \cap \operatorname{Ker}\left(f \circ \varphi^{\sharp}\right)$.

Now by (5) and (7) we have

$$
\begin{aligned}
Y & \cong\left(f \circ \varphi^{\sharp}\right)\left(\delta_{M}^{\#} H^{-1}\left(G, L^{\times} N_{M / L} J_{M} / L^{\times}\right)\right) \\
& =\left(f \circ \delta^{\sharp} \circ \lambda^{\#}\right) H^{-1}\left(G, L^{\times} N_{M / L} J_{M} / L^{\times}\right) \\
& \cong\left(\delta^{\sharp}\left(\operatorname{Im} \lambda^{\#}\right)+\operatorname{Cor}_{H, G}\left(\operatorname{Im} \delta_{H}^{\#}\right)\right) / \operatorname{Cor}_{H, G}\left(\operatorname{Im} \delta_{H}^{\#}\right) \\
& =\left(\delta^{\sharp}\left(\operatorname{Im} \lambda^{\#}\right)+\delta^{\sharp} \operatorname{Cor}_{H, G} H^{-1}\left(H, C_{L}\right)\right) / \delta^{\#} \operatorname{Cor}_{H, G} H^{-1}\left(H, C_{L}\right) \\
& \cong \frac{\operatorname{Im} \lambda^{\#}+\operatorname{Cor}_{H, G} H^{-1}\left(H, C_{L}\right)+\operatorname{Ker} \delta^{\#}}{\operatorname{Cor}_{H, G} H^{-1}\left(H, C_{L}\right)+\operatorname{Ker} \delta^{\#}} .
\end{aligned}
$$

Since $\operatorname{Ker} \delta^{\sharp}=j^{\sharp} H^{-1}\left(G, J_{L}\right)$, it follows from (6), (8) and (9) the following

THEOREM 1. Let $K$ be a Galois extension of $k$ and $M$ be an abelian extension over $K$. Let $L$ be a subfield of $M$ which contains the genus field $K_{M / k}^{*}$ of $K$ in $M$ with respect to $k$. Assume that $L$ is normal over $k$ and put $G=G(L / k)$ and $H=G(L / K)$. Denote by $\hat{L}_{M / k}$ the maximal extension of $L$ which is EL-genus and central with respect to $\dot{k}$. Then we have

$$
G\left(\hat{L}_{M / k} / L\right) \cong \frac{H^{-1}\left(G, C_{L}\right)}{\lambda^{\sharp} H^{-1}(G, D(M / L))+\operatorname{Cor}_{H, G} H^{-1}\left(H, \mathrm{C}_{L}\right)+j^{\sharp} H^{-1}\left(G, J_{L}\right)}
$$


where $D(M / L)$ is the idele class group in $L$ corresponding to $M$ by class field theory; $\lambda^{\#}$ and $j^{\#}$ are induced respectively by the injection map $\lambda$ of $D(M / L)$ to $C_{L}$ and the natural homomorphism $j$ of $J_{L}$ to $C_{L}$.

CoRollary. Let $K$ be a Galois extension of $k$ and $L$ be a subfield of the absolute class field $\bar{K}$ of $K$. Assume that $L$ contains the genus field of $K$ with respect to $k$ and $L$ is normal over $k$. Assume further that any unit of $k$ which is everywhere locally norm from $K$ is a norm of an element of $K$. Put $G=G(L / k), H=G(L / K)$ and let $G_{\nu}$ be the decomposition group of any one of the prime divisors $\mathfrak{p}_{\nu}$ in $L, \mathfrak{p}_{\nu}$ running over all finite and infinite primes of $k$ ramified in $L(\nu=1, \cdots, t)$. Then

$$
\begin{aligned}
G\left(\hat{L}_{\bar{K} / k} / L\right) & \cong \frac{H^{-1}\left(G, C_{L}\right)}{\operatorname{Cor}_{H, G} H^{-1}\left(H, C_{L}\right)+\sum_{\nu=1}^{t} \operatorname{Cor}_{G_{\nu}, G} H^{-1}\left(G_{\nu}, C_{L}\right)} \\
& \cong \frac{H^{-3}(G, Z)}{\operatorname{Cor}_{H, G} H^{-3}(H, Z)+\sum_{\nu=1}^{t} \operatorname{Cor}_{G_{\nu}, G} H^{-3}\left(G_{\nu}, Z\right)} .
\end{aligned}
$$

Proof. By the assumption for the units of $k$, Proposition 5 implies $G\left(\hat{L}_{\bar{K} / k} / L\right) \cong X$, where $X$ is as in (5). Then the corollary follows from (8), since it is well known that $H^{-1}\left(G, C_{L}\right) \cong H^{-3}(G, Z)$ and $j^{\sharp} H^{-1}\left(G, J_{L}\right)$ $\cong \sum_{\nu=1}^{t} \operatorname{Cor}_{G_{\nu}, G} H^{-3}\left(G_{\nu}, Z\right)$.

THEOREM 2. Let $K$ be a Galois extension of $k$ with Galois group $\mathrm{g}$ and let $M$ be an abelian extension over $K$. Denote by $D(M / K)$ the idele class group in $K$ corresponding to $M$ by class field theory. Then we have

$$
G\left(\hat{K}_{M / k} / K_{M / k}^{*}\right) \cong \frac{H^{-1}\left(\mathfrak{g}, C_{K}\right)}{\lambda^{\#} H^{-1}(\mathfrak{g}, D(M / K))+\sum_{\nu=1}^{t} \operatorname{Cor}_{g \nu, g} H^{-1}\left(\mathfrak{g}_{\nu}, C_{K}\right)},
$$

where $\mathfrak{g}_{\nu}$ is the decomposition group of any one of the prime divisors of $\mathfrak{p}_{\nu}$ in $K, \mathfrak{p}_{\nu}$ running over all finite and infinite primes of $k$ ramified in $K$.

Proof. We note that the right hand sides of Proposition 3 and Proposition 4 are coincide, when $L=K$. Theorem 1 was obtained by transforming the right hand side of Proposition 4. Therefore $G\left(\hat{K}_{M / k}\right)$ $K_{M / k}^{*}$ ) is isomorphic to the right hand side of Theorem 1 by putting $L=K$. Since it is well known that $j^{\sharp} H^{-1}\left(\mathfrak{g}, J_{K}\right) \cong \sum_{\nu=1}^{t} \operatorname{Cor}_{g_{\nu}, g} H^{-1}\left(\mathfrak{g}_{\nu}, C_{K}\right)$, the theorem is proved. 
Now for a while let $G$ be any finite group, $H$ be a subgroup of $G$ and $A$ be any $G$-module. Denote by $I_{G}$ the augmentation ideal of the group ring $Z G$. Denote further by $N_{H}$ the trace map, namely $N_{H}(a)=$ $\sum_{\sigma \in H} \sigma a$ for an element $a$ of $A$. Then $N_{H}$ is an endomorphism of $A$. Denote by $O_{H}(A)$ the kernel of $N_{H}$. Then we have the isomorphism $\kappa_{-1}: H^{-1}(G, A) \cong O_{G}(A) / I_{G}(A)$, and $\operatorname{Cor}_{H, G} H^{-1}(H, A) \cong\left(O_{H}(A)+I_{G}(A)\right) / I_{G}(A)$. Hence $H^{-1}(G, A) / \operatorname{Cor}_{H, G} H^{-1}(H, A) \cong O_{G}(A) /\left(O_{H}(A)+I_{G}(A)\right)$. Now assume that $H$ is normal in $G$. Then we see $N_{H} O_{G}(A)=O_{G / H}\left(N_{H} A\right)$ and $N_{H} I_{G}(A)=I_{G}\left(N_{H} A\right)=I_{G / H}\left(N_{H} A\right)$. Hence $H^{-1}(G, A) / \operatorname{Cor}_{H, G} H^{-1}(H, A) \cong$ $O_{G}\left(N_{H} A\right) / I_{G / H}\left(N_{H} A\right) \cong H^{-1}\left(G / H, N_{H} A\right)$. Let $\tilde{N}_{H}$ be the homomorphism induced from $N_{H}$ of $O_{G}(A) / I_{G}(A)$ to $O_{G / H}\left(N_{H} A\right) / I_{G / H}\left(N_{H} A\right)$. Then we have

Proposition 6. Let $G$ be a finite group, $H$ be a normal subgroup of $G$ and $A$ be a G-module. Then we have the following exact sequence:

$$
H^{-1}(H, A) \stackrel{\operatorname{Cor}_{H, G}}{\longrightarrow} H^{-1}(G, A) \stackrel{\widetilde{N}_{H}}{\longrightarrow} H^{-1}\left(G / H, N_{H} A\right) \longrightarrow 0 \text {. }
$$

Now we come back to the investigation of the structure of $G\left(\hat{L}_{M / K} / L\right)$. Notation being as before, we have the following isomorphism by Theorem 1 and Proposition 6.

$$
G\left(\hat{L}_{M / k} / L\right) \cong \frac{\tilde{N}_{H} H^{-1}\left(G, C_{L}\right)}{\tilde{N}_{H} \lambda^{\sharp} H^{-1}(G, D(M / L))+\tilde{N}_{H} j^{\sharp} H^{-1}\left(G, J_{L}\right)} .
$$

We consider the following commutative diagram.

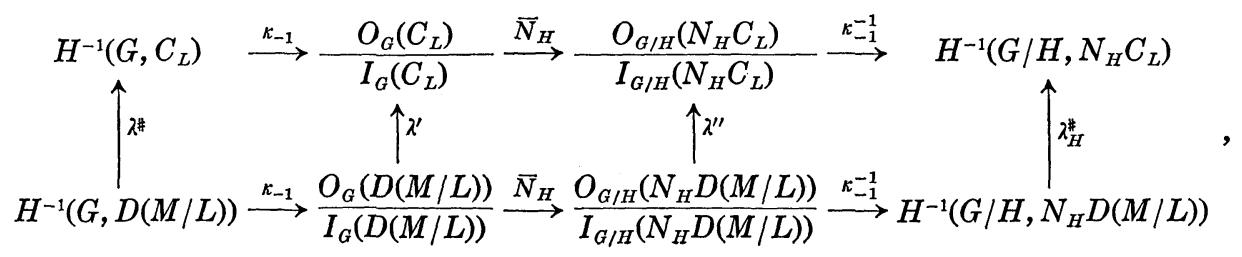

where $\lambda^{\prime}$ and $\lambda^{\prime \prime}$ are induced from the injections of the numerators respectively and $\lambda_{H}^{\#}$ is induced from also the injection map $\lambda_{H}: N_{H} D(M / L)$ $\rightarrow N_{H} C_{L}$. Then the following proposition follows immediately from (10), the above diagram and Proposition 6.

Proposition 7. Notation being as in Theorem 1 and as above, we have 


$$
G\left(\hat{L}_{M / k} / L\right) \cong \frac{H^{-1}\left(G / H, N_{H} C_{L}\right)}{\lambda_{H}^{\#} H^{-1}\left(G / H, N_{H} D(M / L)\right)+\tilde{N}_{H} j^{\sharp} H^{-1}\left(G, J_{L}\right)} .
$$

\section{§4. Cohomological expression of $G\left(K^{(i+1)} / K^{(i)}\right)$}

Let $K$ be a Galois extension of $k$ with Galois group $g$ and $M$ be a class field over $K$ corresponding to an idele class group $D(M / K)$. Denoting by $C_{K}$ the idele class group of $K$ as before, put $\mathfrak{D}=C_{K} / D(M / K)$. Assume that ${ }^{8)} M$ is an $E L$-genus extension of $K$ with respect to $k$ and normal over $k$. Then $\mathfrak{D}$ is a $g$-module in natural way. Let $\mathfrak{D}^{(i)}, i=1$, $2, \cdots$, be the lower central series for $\mathfrak{D}$ with respect to $g$ in the sense of Introduction and let $K_{M / k}^{(i)}$ be the extension of $K$ corresponding to $\mathfrak{D}^{(i)}$. Then $D\left(K_{M / k}^{(i)} / K\right)=\left(I_{\mathrm{g}}^{i} J_{K} \cdot K^{\times} \cdot N_{M / K} J_{M}\right) / K^{\times}$and $K_{M / k}^{(1)}=\hat{K}_{M / k}$. We call the field $K_{M / k}^{(i)}$ the $i$-th central class field of $K$ in $M$ with respect to $k$. When $M$ is equal to the absolute class field, we call $K_{M / k}^{\langle i\rangle}$ simply the $i$-th central class field of $K$ with respect to $k$ and denote it by $K^{(i)}$.

Now since $\mathfrak{D}^{(i)}=I_{g}^{i} \mathfrak{D}$ by definition, we have for $i \geqq 1$

$$
G\left(K_{M / k}^{(i+1)} / K_{M / k}^{(i)}\right) \cong I_{\mathrm{g}}^{i \mathfrak{D}} / I_{\mathfrak{g}}^{i+1} \mathfrak{S} \cong H^{-1}\left(\mathfrak{g}, I_{\mathrm{g}}^{i \mathfrak{D})}\right.
$$

For the sake of simplicity, denote by $C^{(i)}$ the idele class group of $K_{M / k}^{(i)}$ and put $H_{i}=G\left(K_{M / k}^{(i)} / K\right)$. Then $D\left(K_{M / k}^{(i)} / K\right)=N_{H_{i}} C^{(i)}$ and we have the following exact sequence in natural way:

$$
0 \longrightarrow D(M / K) \stackrel{\lambda}{\longrightarrow} D\left(K_{M / k}^{(i)} / K\right) \stackrel{\mu}{\longrightarrow} \mathfrak{D}^{(i)} \longrightarrow 0 .
$$

This implies the following cohomology exact sequence:

$$
\begin{aligned}
\cdots \longrightarrow & H^{-1}(\mathfrak{g}, D(M / K)) \stackrel{\lambda_{-1}^{\#}}{\longrightarrow} H^{-1}\left(\mathfrak{g}, N_{H_{i}} C^{(i)}\right) \stackrel{\mu_{-1}^{\#}}{\longrightarrow} H^{-1}\left(\mathfrak{g}, \mathfrak{D}^{(i)}\right) \\
& \stackrel{\delta^{\sharp}}{\longrightarrow} H^{0}(\mathfrak{g}, D(M / K)) \stackrel{\lambda_{0}^{\#}}{\longrightarrow} H^{0}\left(\mathfrak{g}, N_{H_{i}} C^{(i)}\right) \stackrel{\mu_{0}^{\#}}{\longrightarrow} \cdots .
\end{aligned}
$$

Hence we have

$$
\begin{aligned}
{\left[H^{-1}\left(\mathfrak{g}, N_{H_{i}} C^{(i)}\right): \lambda_{-1}^{\#} H^{-1}(\mathfrak{g}, D(M / K)]\right.} & \leqq\left|H^{-1}\left(\mathfrak{g}, \mathfrak{D}^{(i)}\right)\right| \\
& =\left|G\left(K_{M / k}^{(i+1)} / K_{M / k}^{(i)}\right)\right| .
\end{aligned}
$$

On the other hand if we put $L=K_{M / k}^{(i)}$ in Proposition 7 , then $\hat{L}_{M / k}=$ $K_{M / k}^{(i+1)}$ and we have the opposite inequality to (11). Therefore in the above cohomology exact sequence $\mu_{-1}^{*}$ is surjective and we have the

8) Since we treat only EL-genus extensions contained in $M$, we add this assumption for the sake of simplicity. Cf. also the footnote 5 ). 
following

THEOREM 3. Let $K$ be a Galois extension of $k$ with Galois group $\mathfrak{g}$ and let $M$ be an EL-genus extension over $K$ with respect to $k$, abelian over $K$ and normal over $k$. Then for $i \geqq 1$ we have

$$
G\left(K_{M / k}^{(i+1)} / K_{M / k}^{(i)}\right) \cong H^{-1}\left(\mathrm{~g}, D\left(K_{M / k}^{(i)} / K\right)\right) / \lambda^{\sharp} H^{-1}(\mathrm{~g}, D(M / K)),
$$

where $\lambda^{\#}$ is induced from the injection map $\lambda$ of the idele class group $D(M / K)$ to $D\left(K_{M / k}^{(i)} / K\right)$.

We proceed our discussion to express the right hand side of Theorem 3 by (-2)-cohomology groups. Notation being as above, put $D^{(i)}=$ $D\left(K_{M / k}^{(i)} / K\right)$ and consider the following natural commutative sequence:

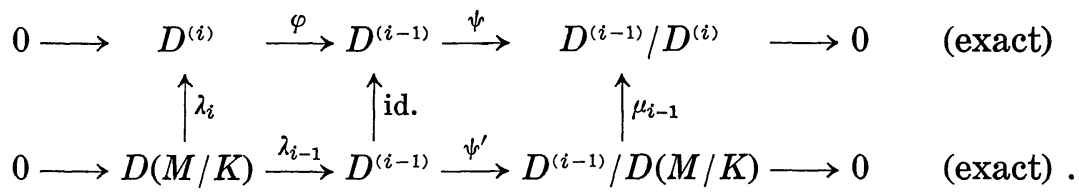

Then we have the following commutative diagram:

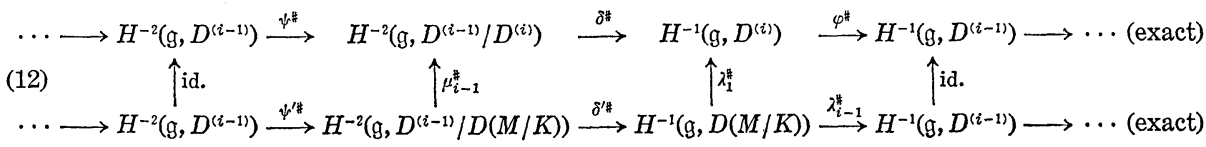

We have

$$
\frac{\varphi^{\sharp} H^{-1}\left(\mathrm{~g}, D^{(i)}\right.}{\lambda_{i-1}^{\#} H^{-1}(\mathrm{~g}, D(M / K))}=0
$$

In fact the left hand side of (13) is isomorphic to

$$
\frac{H^{-1}\left(\mathfrak{g}, D^{(i-1)}\right) / \lambda_{i-1}^{\#} H^{-1}(\mathfrak{g}, D(M / K))}{H^{-1}\left(\mathfrak{g}, D^{(i-1)}\right) / \varphi^{\sharp} H^{-1}\left(\mathfrak{g}, D^{(i)}\right)}
$$

and Theorem 3 implies $H^{-1}\left(\mathfrak{g}, D^{(i-1)}\right) / \lambda_{i-1}^{\#} H^{-1}(\mathfrak{g}, D(M / K)) \cong G\left(K_{M / k}^{(i)} / K_{M / k}^{(i-1)}\right)$. Moreover put $M^{\prime}=K_{M / k}^{(i)}$. Then $K_{M^{\prime} / k}^{(i-1)}=K_{M / k}^{(i-1)}, K_{M^{\prime} / k}^{(i)}=M^{\prime}$ and $D\left(M^{\prime} / K\right)$ $=D^{(i)}$. Hence Theorem 3 implies $H^{-1}\left(\mathfrak{g}, D^{(i-1)}\right) / \varphi^{\sharp} H^{-1}\left(\mathfrak{g}, D^{(i)}\right) \cong G\left(K_{M / k}^{(i)} /\right.$ $K_{M / k}^{(i-1)}$ ). Thus (13) is proved.

LEMMA. Let the following diagram of modules is commutative: 


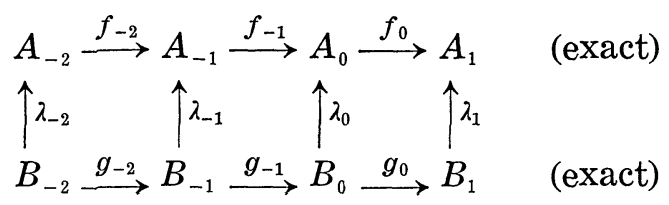

Suppose that $\lambda_{-2}$ and $\lambda_{1}$ implies $A_{-2} \cong B_{-2}$ and $A_{1} \cong B_{1}$ respectively. Then $A_{0} / \lambda_{0} B_{0}$ is a group extension of $f_{0} A_{0} / \lambda_{1} g_{0} B_{0}$ with kernel $A_{-1} / \lambda_{-1} B_{-1}$.

Proof. It is obvious that $A_{0} / \lambda_{0} B_{0}$ is a group extension of $A_{0} /$ $\left(\lambda_{0} B_{0}+f_{-1} A_{-1}\right)$ with kernel $\left(\lambda_{0} B_{0}+f_{-1} A_{-1}\right) / \lambda_{0} B_{0}$. Furthermore we have

$$
\frac{A_{0}}{\lambda_{0} B_{0}+f_{-1} A_{-1}} \cong \frac{A_{0} / f_{-1} A_{-1}}{\left(\lambda_{0} B_{0}+f_{-1} A_{-1}\right) / f_{-1} A_{-1}} \cong \frac{f_{0} A_{0}}{f_{0} \lambda_{0} B_{0}}=\frac{f_{0} A_{0}}{\lambda_{1} g_{0} B_{0}}
$$

and

$$
\begin{aligned}
\frac{\lambda_{0} B_{0}+f_{-1} A_{-1}}{\lambda_{0} B_{0}} & \cong \frac{f_{-1} A_{-1}}{\lambda_{0} B_{0} \cap f_{-1} A_{-1}}=\frac{f_{-1} A_{-1}}{\lambda_{0} B_{0} \cap \operatorname{Ker} f_{0}}=\frac{f_{-1} A_{-1}}{\lambda_{0}\left(\operatorname{Ker}\left(f_{0} \lambda_{0}\right)\right)} \\
& =\frac{f_{-1} A_{-1}}{\lambda_{0}\left(\operatorname{Ker} g_{0}\right)}=\frac{f_{-1} A_{-1}}{\lambda_{0}\left(g_{-1} B_{-1}\right)}=\frac{f_{-1} A_{-1}}{f_{-1} \lambda_{-1} B_{-1}} \cong \frac{A_{-1}}{\lambda_{-1} B_{-1}},
\end{aligned}
$$

since $\lambda_{-1} B_{-1} \supset \lambda_{-1} g_{-2} B_{-2}=f_{-2} A_{-2}=\operatorname{Ker} f_{-1}$.

Now the following theorem follows immediately from (12), (13), lemma and Theorem 3.

THEOREM 4. Let $K$ be a Galois extension of $k$ with Galois group $\mathfrak{g}$ and let $M$ be an abelian extension of $K$ which is normal over $k$ and $E L$ genus over $K$ with respect to $k$. Then for $i \geqq 1$ we have

$$
G\left(K_{M / k}^{(i+1)} / K_{M / k}^{(i)}\right) \cong \frac{H^{-2}\left(\mathfrak{g}, D\left(K_{M / k}^{(i-1)} / K\right) / D\left(K_{M / k}^{(i)} / K\right)\right)}{\mu_{2-1}^{\#} H^{-2}\left(\mathfrak{g}, D\left(K_{M / k}^{(i-1)} / K\right) / D(M / K)\right)},
$$

where $\mu_{i-1}^{*}$ is induced from the natural homomorphism $\mu_{i-1}$ of $D\left(K_{M / k}^{(i-1)} / K\right) / D(M / K)$ to $D\left(K_{M / k}^{(i-1)} / K\right) / D\left(K_{M / k}^{(i)} / K\right)$.

\section{§5. Reduction formula for $G\left(K_{M / k}^{(i+1)} / K_{M / k}^{(i)}\right)$}

Let Notation and assumption be as in Theorem 4 and for the sake of simplicity put $K^{(i)}=K_{M / k}^{(i)}$ and $D^{(i)}=D\left(K_{M / k}^{(i)} / K\right)$. Especially $K^{(0)}=K$, $K^{(1)}=\hat{K}_{M / k}$ and $D^{(0)}=C_{K}$.

For $i \geqq 1$, Theorem 4 shows that $G\left(K^{(i+1)} / K^{(i)}\right)$ is isomorphic to a homomorphic image of $H^{-2}\left(g, G\left(K^{(i)} / K^{(i-1)}\right)\right)$. We study the homomorphism explicitly. Put $\mathfrak{g}_{0}=\mathfrak{g} /[\mathfrak{g}, \mathfrak{g}]$, where $[\mathfrak{g}, \mathfrak{g}]$ is the commutator subgroup of 
g. Let $\alpha=\sum_{\bar{\tau} \in g_{0}} \bar{\tau} \otimes \overline{\mathfrak{a}}_{\bar{\tau}} \in \mathfrak{g}_{0} \otimes D^{(i-1)} / D^{(i)}$, where $\tau \in \mathfrak{g}, \mathfrak{a}_{\bar{\tau}} \in D^{(i-1)}$ and the bar means the class in obvious manner. Then since $D^{(i-1)} / D^{(i)}$ is g-invariant, the isomorphism

$$
\theta_{i} ; \mathfrak{g}_{0} \otimes D^{(i-1)} / D^{(i)} \rightarrow H^{-2}\left(\mathfrak{g}, D^{(i-1)} / D^{(i)}\right)
$$

is defined by $\theta_{i}(\alpha) \equiv \sum_{\bar{\tau} \in g_{0}} \overline{\mathfrak{a}}_{\bar{\tau}} *[\tau] \bmod$. coboundary, where the standard expression of $(-2)$-cocycles follows Babakhanian $[1, \S 21]$.

We have now the composition of the homomorphisms

$$
\Theta_{i}: \mathfrak{g}_{0} \otimes D^{(i-1)} / D^{(i)} \stackrel{\theta_{i}}{\longrightarrow} H^{-2}\left(\mathfrak{g}, D^{(i-1)} / D^{(i)}\right) \stackrel{\delta^{\sharp}}{\longrightarrow} H^{-1}\left(\mathfrak{g}, D^{(i)}\right) \stackrel{\kappa_{-1}}{\longrightarrow} D^{(i)} / D^{(i+1)},
$$

where $\delta^{\sharp}$ is the same as in (12). Then for $\alpha$ as above we have $e^{9)}$

$$
\begin{aligned}
\Theta_{i}(\alpha) & \equiv \kappa_{-1}\left(\left(\sum_{\bar{\tau} \in \mathbb{8}_{0}}\left(\tau^{-1}-1\right) \mathfrak{a}_{\bar{\tau}}\right) *[]\right) \\
& \equiv \sum_{\bar{\tau} \in \mathbb{g}_{0}}\left(\tau^{-1}-1\right) \mathfrak{a}_{\bar{\tau}} \quad \bmod . D^{(i+1)} .
\end{aligned}
$$

It follows from Theorem 4 that $G\left(K^{(i+1)} / K^{(i)}\right) \cong D^{(i)} / D^{(i+1)} \cong$ $H^{-2}\left(\mathrm{~g}, D^{(i-1)} / D^{(i)}\right) / \mu_{i}^{*} H^{-2}\left(\mathrm{~g}, D^{(i-1)} / D(M / K)\right)$. This implies that $\Theta_{i}$ is surjective and we have ${ }^{10)}$

$$
\begin{aligned}
\operatorname{Ker} \Theta_{i} & =\theta_{i}^{-1} \mu_{i}^{\sharp} H^{-2}\left(\mathfrak{g}, D^{(i-1)} / D(M / K)\right) \\
& =\left\{\sum_{\bar{\tau} \in \mathfrak{g}_{0}} \bar{\tau} \otimes \overline{\mathfrak{a}}_{\bar{\tau}} \mid \overline{\mathfrak{a}}_{\overline{\mathfrak{\tau}}}=\sum_{\rho \in \bar{\tau}} \overline{\mathfrak{b}}_{\rho}, \mathfrak{b}_{\rho} \in D^{(i-1)}, \sum_{\rho \in \mathfrak{g}}\left(\rho^{-1}-1\right) \mathfrak{b}_{\rho} \in D(M / K)\right\} .
\end{aligned}
$$

Denote by $\mathrm{g}_{0}^{(r)}$ the tensor product of $r$-copies of $\mathfrak{g}_{0}$. Moreover denote by $D^{(r)}\left(\mathfrak{g}, M, K^{(i)} / K\right)$, for $i \geqq 0$ and $r \geqq 1$, the subgroup of $\mathfrak{g}_{0}^{(r)} \otimes D^{(i)} / D^{(i+1)}$ which consists of

$$
\sum_{\overline{\tau^{(1)}}, \ldots, \overline{\tau^{(r)} \in g_{0}}} \overline{\tau^{(1)}} \otimes \cdots \otimes \overline{\tau^{(r)}} \otimes \bar{a}\left(\overline{\tau^{(1)}}, \cdots, \overline{\tau^{(r)}}\right),
$$

where $\overline{\mathfrak{a}}\left(\overline{\tau^{(1)}}, \cdots, \overline{\left.\tau^{(r)}\right)}=\sum_{\substack{\rho_{i} \in r^{(i)} \\ i=1, \cdots, r}} \overline{\mathfrak{b}}\left(\rho_{1}, \cdots, \rho_{r}\right)\right.$ and $\overline{\mathfrak{b}}\left(\rho_{1}, \cdots, \rho_{r}\right)$ is the class of $D^{(i)} / D^{(i+1)}$ represented by $\mathfrak{b}\left(\rho_{1}, \cdots, \rho_{r}\right)$ of $D^{(i)}$ which satisfies

$$
\sum_{\rho_{1}, \cdots, \rho_{r} \in \mathbb{B}}\left(\rho_{1}-1\right) \cdots\left(\rho_{r}-1\right) \mathfrak{b}\left(\rho_{1}, \cdots, \rho_{r}\right) \in D(M / K) .
$$

For $i=0$, put $D^{(r)}(\mathfrak{g}, M, K)=D^{(r)}\left(\mathfrak{g}, M, K^{(0)} / K\right)$.

Then we have the following main theorem.

9) We use the additive expression for the product in $C_{K}$.

10) Cf. Babakhanian [1, §21.2]. 
THEOREM 5. Let $K$ be a Galois extension of $k$ with Galois group $g$ and let $M$ be an abelian extension of $K$ which is normal over $k$ and EL-genus with respect to $k$. Then notation being as above we have

$$
G\left(K_{M / k}^{(i+1)} / K_{M / k}^{(i)}\right) \cong \frac{\mathfrak{g}_{0}^{(i)} \otimes C_{K} / D\left(\hat{K}_{M / k} / K\right)}{D^{(i)}(\mathfrak{g}, M, K)}
$$

Proof. By (16) and the definition of $D^{(r)}\left(\mathfrak{g}, M, K^{(i)} / K\right)$, we have $\operatorname{Ker} \Theta_{i}=D^{(1)}\left(\mathfrak{g}, M, K^{(i-1)} / K\right)$ and further

$$
G\left(K^{(i+1)} / K^{(i)}\right) \cong \frac{\mathfrak{g}_{0} \otimes D^{(i-1)} / D^{(i)}}{D^{(1)}\left(\mathrm{g}, M, K^{(i-1)} / K\right)}
$$

For $i \geqq 0$ and $r \geqq 1$ put $\Theta_{i}^{(r)}=1_{g(r-1)} \otimes \Theta_{i}$, which is a surjective homomorphism from $\mathfrak{g}_{0}^{(r)} \otimes D^{(i-1)} / D^{(i)}=\mathfrak{g}_{0}^{(r-1)} \otimes \mathfrak{g}_{0} \otimes D^{(i-1)} / D^{(i)}$ to $\mathfrak{g}_{0}^{(r-1)} \otimes D^{(i)} / D^{(i+1)}$. Then it is easy to see that $\Theta_{i}^{(r)} D^{(r)}\left(\mathfrak{g}, M, K^{(i-1)} / K\right)=D^{(r-1)}\left(\mathfrak{g}, M, K^{(i)} / K\right)$ and $\operatorname{Ker} \Theta_{i}^{(r)}=\mathfrak{g}_{0}^{(r-1)} \otimes \operatorname{Ker} \Theta_{i} \subset D^{(r)}\left(\mathfrak{g}, M, K^{(i-1)} / K\right)$. Hence $\Theta_{i}^{(r)}$ implies

$$
\frac{\mathfrak{g}_{0}^{(r)} \otimes D^{(i-1)} / D^{(i)}}{D^{(r)}\left(\mathfrak{g}, M, K^{(i-1)} / K\right)} \cong \frac{\mathfrak{g}_{0}^{(r-1)} \otimes D^{(i)} / D^{(i+1)}}{D^{(r-1)}\left(\mathfrak{g}, M, K^{(i)} / K\right)}
$$

Now by applying this reduction formula to (18) repeatedly, the theorem is proved.

Remark. If $K$ is cyclic over $k$ and the Galois group is generated by $\sigma$, then $\hat{K}_{M / k}=K_{M / k}^{*}$ and $g_{0}^{(i)} \otimes C_{K} / D\left(\hat{K}_{M / k} / K\right) \cong C_{K} / D\left(K_{M / k}^{*} / K\right)$. Moreover easily $D^{(i)}(\mathfrak{g}, M, K)=\left\{\mathfrak{a} \bmod . D\left(K_{M / k}^{*} / K\right) \mid \mathfrak{a} \in C_{K}, \mathfrak{a}^{(\sigma-1)^{i}} \in D(M / K)\right\}$. Therefore Theorem 5 coincides with (1) in $\S 1$, when $K$ is cyclic over $k$.

\section{REFERENCES}

[1] A. Babakhanian, Cohomological methods in group theory, Marcel Dekker, Inc., New York (1972).

[2] P. Barrucand and H. Cohn, Note on primes of type $x^{2}+32 y^{2}$, class number, and residuacity, J. Reine Angew, Math., 238 (1969), 67-70.

[ 3 ] A. Fröhlich, The genus field and genus group in finite number fields I, II, Mathematika, 6 (1959), 40-46, 142-146.

[ 4 ] Y. Furuta, The genus field and genus number in algebraic number fields, Nagoya Math. J., 29 (1967), 281-285.

[5] Y. Furuta, Über die zentrale Klassenzahl eines relativ-galoisschen Zahlkörpers, J. Number Theory, 3 (1971), 318-322.

[6] Y. Furuta, On class field towers and the rank of ideal class groups, Nagoya Math. J., 48 (1972), 147-157.

[7] G. Gras, Sur le -groupe des classes des extensions cycliques de degré premier $\ell$, C. R. Acad. Sci. Paris, 274 (1972), 1145-1148. 
[ 8 ] G. Gras, Sur le $\ell$-classes d'ideaux dans les extensions cycliques relatives de degré premier $\ell$, Ann. Inst. Fourier, Grenoble, 23,3 (1973), 1-48; 23,4 (1973), 1-44.

[ 9 ] H. Hasse, Über die Klassenzahl des Körpers $P(\sqrt{-p})$ mit einer Primzahl $p \equiv 1$ mod. $2^{3}$, Aequations Mathematicae, 3 (1969), 165-169.

[10] H. Hasse, Über die Teilbarkeit durch $2^{3}$ der Klassenzahl der quadratischen Zahlkörper mit genau zwei verschiedenen Diskriminantenprimteilern, Math. Nachr., 46 (1970), 61-70.

[11] K. Masuda, An application of the generalized norm residue symbol, Proc. Amer. Math. Soc., 10 (1959), 245-252.

Kanazawa University 